\title{
SUPERFICIAL HERPETIC KERATITIS TREATED WITH 5-IODO-2'-DEOXYURIDINE*
}

\author{
BY \\ N. N. SOOD † AND V. J. MARMION \\ St. Paul's Eye Hospital, Liverpool
}

THE results of treating herpetic keratitis with the antiviral agent 5-iodo-2'deoxyuridine have proved, after initial enthusiasm, to be somewhat variable. In most instances the number of cases analysed has been small (Bennett, 1962; Corrigan, Gilkes, and Roberts, 1962; Davidson, 1962; Kaufman and Maloney, 1962; Luntz and MacCallum, 1963). More accurate conclusions can best be reached by a doubleblind trial (Patterson, Fox, Davies, Maguire, Sellors, Wright, Rice, Cobb, and Jones, 1963) and the evaluation of a large series of patients. That IDU is effective cannot be seriously doubted, but its comparative value and its toxicity must be watched.

For these reasons it was decided to analyse the results of a large series and in particular to look for the causes of failure.

\section{Material and Methods}

150 unselected patients attending the Out-Patients' Department of St. Paul's Eye Hospital during the period January to July, 1963, were treated with 0.1 per cent. IDU drops hourly by day and 2-hourly at night. When the ointment was available it was used 3-hourly at night. All the patients were asked to keep a record of instillations. At the first visit a slit-lamp examination was carried out. The site and size of the lesion and all relevant information, such as previous attacks, etc., were recorded on a special form. The patients were re-examined on the third day and the prognosis was based on the degree of fragmentation of the lesion (at least one-third) and the absence of any overall increase in the size of the dendritic ulcer. The patients were seen every 2 to 3 days and the size of the lesion was delineated, on each occasion, with fluorescein 2 per cent. and counterstained with rose Bengal 1 per cent. The condition was regarded as cured from the time when no staining occurred with either stain. Treatment was continued until the fifth day, and if no further improvement had been obtained the case was regarded as a failure and the ulcer was iodized. The patients were followed for at least one week after the corneal staining had ceased.

The site of the lesion on the cornea was described as central (C), paracentral (PC), or limbal (L) as indicated in Fig. 1. The black central area represents the normal pupillary area.

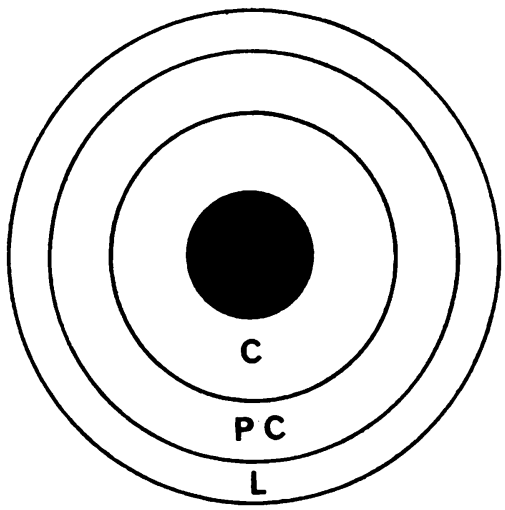

FIG. 1.-Diagram of cornea, showing (C) central area; (PC) paracentral area; and (L) limbal area.

C Diameter of $6.5 \mathrm{~mm}$. from centre of cornea.

PC Area 6.5 to $9.5 \mathrm{~mm}$. from centre of cornea.

L Area $9.5 \mathrm{~mm}$. and over from centre of cornea.

* Received for publication January 2, 1964.

† Present address: Dept. of Ophthalmology, Medical College Hospital, Pondicherry.

$\ddagger$ Present address: Bristol Eye Hospital, Bristol. 
The size of the lesions was not calculated in millimetres but was estimated as a proportion of the total corneal area. Those extending to a quarter or less were graded as small, and those extending to more than a quarter as large. Corneal sensation was measured throughout with a thin wisp of cotton wool.

Herpetic keratitis tends to become chronic, so that the duration of the disease before the first hospital visit was variable. The duration was measured by the history of pain, photophobia, and lacrimation.

\section{Results}

The results show that IDU is of limited value in the treatment in herpetic (dendritic) keratitis.

Table I indicates the sex, side affected, site, and success and failure rates.

TABLE I

DISTRIBUTION OF LESIONS, BY SEX, SIDE AFFECTED, SITE, AND RESULT

\begin{tabular}{|c|c|c|c|c|}
\hline \multirow{2}{*}{ Sex } & \multirow{2}{*}{ Side Affected } & \multirow{2}{*}{ Site } & \multicolumn{2}{|c|}{ Result } \\
\hline & & & Success & Failure \\
\hline \multirow{4}{*}{97 Male } & \multirow{2}{*}{49 Right } & $36 \mathrm{C}$ & 26 & 10 \\
\hline & & $13 \mathrm{~L}$ & 5 & 8 \\
\hline & \multirow{2}{*}{48 Left } & $34 \mathrm{C}$ & 29 & 5 \\
\hline & & $14 \mathrm{~L}$ & 6 & 8 \\
\hline \multirow{4}{*}{53 Female } & \multirow{2}{*}{18 Right } & $10 \mathrm{C}$ & 9 & 1 \\
\hline & & $8 \mathrm{~L}$ & 3 & 5 \\
\hline & \multirow{2}{*}{35 Left } & $23 \mathrm{C}$ & 20 & 3 \\
\hline & & $12 \mathrm{~L}$ & 4 & 8 \\
\hline Total & \multicolumn{2}{|c|}{150} & 102 & 48 \\
\hline
\end{tabular}

Fig. 2 (opposite) shows the duration of the disease as far as could be assessed by the history, and Fig. 3 (opposite) gives a further analysis of Fig. 2 to show the incidence of fresh cases and those in which the same cornea had been involved on a previous occasion. These were further analysed to see if any significant statistical difference existed between the new and the old central lesions in their response to IDU. None was found. In four cases a satisfactory assessment of the duration could not be made and these are excluded from Figs 2 and 3.

The principal factors taken into consideration in evaluating the reasons for failure were site, size, duration, previous steroid administration, incidence of complicating deep keratitis, and early recurrences. The failures were further divided into $(a)$ progressive, (b) static, (c) and recalcitrant (Fig. 4, opposite).

Recurrences were observed in seventeen patients and in twelve they occurred soon after treatment. Six of these responded to IDU initially and only one failed to respond to IDU on the second occasion. Three of the remainder responded to IDU on the second occasion having failed at first. 


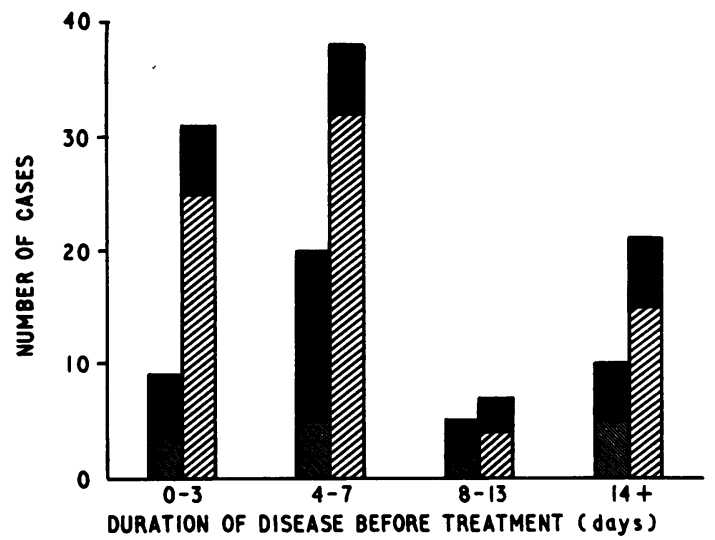

Fig. 2.-Success and failure, analysed by site of lesion and duration of disease before treatment.

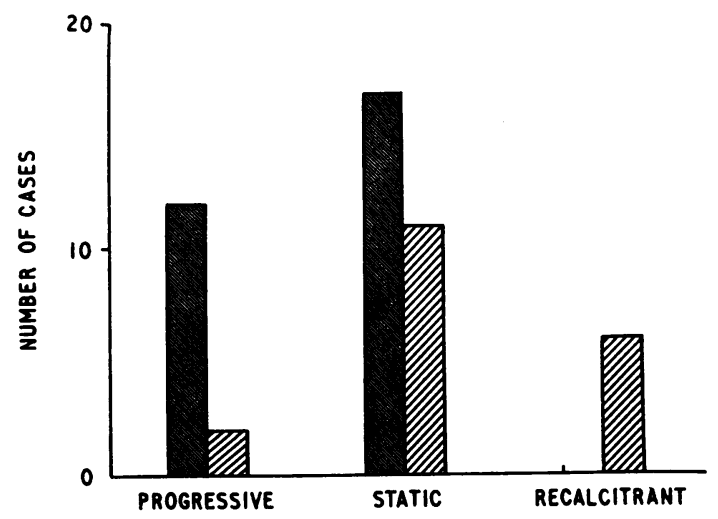

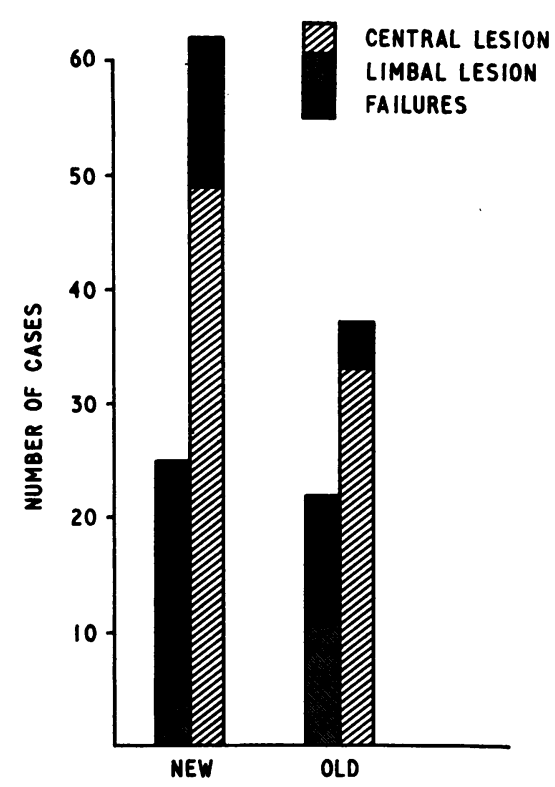

Fig. 3.-Success and failure, analysed by site of lesion and whether it was old or new.

FIG. 4.-Failures analysed by site of lesion and response to treatment.

\section{Discussion}

A review of the cases examined shows that the population sample involved corresponds in age and sex distribution (Table II) to previous large series (Gundersen, 1936; Thygeson, Kimura, and Hogan, 1956).

TABLE II

DISTRIBUTION OF PATIENTS, BY AGE AND SEX

\begin{tabular}{c|c|c}
\hline \multirow{2}{*}{ Age (yrs) } & \multicolumn{2}{|c}{ Sex } \\
\cline { 2 - 3 } & Male & Female \\
\hline $0-9$ & 5 & 10 \\
$10-19$ & 3 & 3 \\
$20-29$ & 7 & 2 \\
$30-39$ & 16 & 7 \\
$40-49$ & 18 & 8 \\
$50-59$ & 30 & 9 \\
$60+$ & 18 & 53 \\
\hline
\end{tabular}


The term "bilateral" was used in our series to describe cases in which the condition occurred in both eyes simultaneously. Three such bilateral cases were encountered; this represents a slightly greater frequency than that reported by Gundersen (1936) but less than that of Thygeson and others (1956). The relatively low incidence of bilateral disease in this or any other series would tend to discount fomites as a means of transmission. The equal distribution between each eye would suggest direct autogenous transmission, probably by fingers. Most patients gave a history of previous labial herpes and this is probably the reservoir of infection. Skin herpes affecting the lid margin was a relatively rare phenomenon.

Ten patients showed two separate dendritic lesions on the cornea. These were not ulcers which were likely to coalesce. All had active labial herpes and all but one responded well to IDU; one of the seventeen recurrences was from this group.

The less frequent occurrence of the condition in women and children is difficult to explain; perhaps the industrious male has more occasion to rub his eyes. Minor or major trauma to the eyes, given a reasonable interval of time for incubation, is a certain trigger mechanism in dendritic ulceration.

Corneal sensation was depressed in all but a very few patients, hypaesthesia being more marked in some than in others. An improvement in sensation was observed in some of the first patients in the series who returned for follow-up towards the end of the trial.

Deep keratitis occurred during the course of treatment in ten patients, six with central and four with limbal lesions. This incidence is lower than that previously reported and somewhat lower than that in cases treated with cauterization. Three of these patients had previously received steroid treatment. Three of them failed to respond to IDU. The average duration of disease was greater in these patients than in the rest of the series.

In nine patients it was possible to establish that local steroid treatment had been given before their attendance at the hospital; in these the failure rate was the same as in the series as a whole, but there was a higher incidence of deep keratitis $(33.3$ per cent.). The steroids had been stopped in all instances before treatment with IDU was started. This higher incidence of deep keratitis could perhaps be related to the suppression of a normal protective mechanism which allowed virus particles to permeate the corneal stroma.

One instance of spontaneous resolution was observed. Though this may occur in up to 10 per cent. (Thygeson and others, 1956), it is rare enough to be discounted in the evaluation of treatment in this dangerous disease, and furthermore, in this case a relapse occurred 10 days later with typical dendritic ulceration.

Our results show that there was a good response to IDU, 68 per cent. having healed within 7 days. One noticeable feature is the comfort afforded by the drops; although administration has to be frequent few patients complained of this inconvenience. Invariably the degree of ciliary injection was reduced and in most instances, even in those who did not respond, a "quiet eye" was observed.

The duration of the disease before treatment did not appear to affect the ultimate outcome; Fig. 2 shows that limbal lesions failed to respond to IDU, and suggests that the drug is more effective in patients who have had the disease for between 4 
and 7 days. This could be explained on the basis of a susceptible phase in the life cycle of the virus when it is neither proliferating nor static.

The mode of action of IDU is not yet clearly known. It may be virocidal or virostatic. The observation that several cases which did not respond in the first instance responded to a second course of IDU favours the latter. Furthermore, Fig. 2 shows little difference in the results according to the phase of the disease. If IDU is virocidal it should be more active in the phase of proliferation (days $0-3$ ); if it is virostatic it should be equally active in 0-3 days as in 4-7 days. The slow rate of healing, taking 3 to 5 days to heal completely, indicates that the drug eliminates virus growth and promotes excretion, probably by killing the cell as well. The latter may account for the large number of indolent ulcers in this series; the seventeen recurrences indicate that virus particles must still remain in the epithelium.

\section{Analysis of Failure Rate}

Previous workers have not commented upon the incidence of limbal dendritic ulcers, but Gundersen (1936) remarked on their poor response to iodization. Our experience was similar right from the start of this trial. The failure rate was higher with the limbal lesions (significant at a level of $P=0.05$ ) which showed a greater tendency to progress, irrespective of the size of the ulcer.

It would be expected that the tear film with its greater volume of the drug would bring a better supply to the limbal regions and thus enhance its action at the limbus. This anomaly may be explained by the better blood supply for detoxication at the limbus and at the lid margin.

It would seem, therefore, that the ideal situation for the successful use of IDU is a central corneal ulcer of short duration which has not previously been treated with steroids, and in which there is no deep keratitis.

The prognosis made on the third day of treatment was of great value. A good prognosis was made for those showing good fragmentation; and a poor one for those showing extension of the lesion or failure to fragment in less than one-third of the lesion. The prognosis thus assigned was correct in all but three instances $(98$ per cent.), and thus provides an accurate estimate, after 3 days of therapy, of the efficacy of the drug. This is of particular importance where deep keratitis supervenes, or where there are other complicating factors.

\section{Toxicity}

Toxic side-effects consisted principally of superficial punctate keratitis which disappeared quite satisfactorily on cessation of the drops. Eighteen cases of indolent ulceration were ascribed to inhibition of epithelialization by the drug; these lesions tended to progress very slowly to healing and showed no active increase in size. Thirdly, conjunctival allergy was attributed to the IDU drops on two occasions.

\section{Summary}

A review of 150 cases of superficial herpetic (dendritic) keratitis is presented. The response to the topical administration of IDU is evaluated and the importance of the high failure rate in limbal lesions is stressed. The value of a prognosis made on the 
third day of treatment is suggested. A note is added on the toxic side-effects of the drug.

Our thanks are due to the surgeons of St. Paul's Eye Hospital, Liverpool, for access to their patients, and to Messrs. Smith Kline and French, Ltd. and Genatosan Ltd. for supplies of the drug for clinical trial.

BennetT, F. (1962). Lancet, 2, 726.

\section{REFERENCES}

Corrigan, M. J., Gilkes, M. J., and Roberts, D. St. Clair (1962). Brit. med. J., $2,304$.

DAVIDSON, S. I. (1962). Lancet, 2, 1326.

Gundersen, T. (1936). Arch. Ophthal. (Chicago), 15, 225.

Kaufman, H. E., and Maloney, E. D. (1962). Ibid., 68, 396.

LuntZ, M. H., and MacCallum, F. D. (1963). Brit. J. Ophthal., 47, 449.

Patterson, A., Fox, A. D., Davies, G., Maguire, C., Holmes Sellors, P. J., Wright, P., Rice, N. S. C., CoBB, B., and Jones, B. R. (1963). Trans. ophthal. Soc. U.K., 83, 583.

Thygeson, P., Kimura, S. J., and Hogan, M. J. (1956). A.M.A. Arch. Ophthal., 56, 375. 